\title{
MIMR A CROSS-COUNTRY SYSTEMATIC LITERATURE REVIEW ON YOUNG ADULTS'KNOWLEDGE AND AWARENESS ON HIV/AIDS
}

\author{
Gilbert Anthony O. Abaño \\ Faculty of Education, Eastern Visayas State University- Ormoc City Campus \\ "Corresponding author's email: gilvert22@gmail.com
}

\begin{abstract}
With the aim to better appreciate the young adults' knowledge and degree of awareness on global issue of HIV/AIDS, the researcher purposively reviewed 25 qualified documents, utilized data search engine such as ProQuest, CINAHL, PubMed and Ebscohost to consolidate literature findings from 2012-2017. The researcher used content analysis technique to screen and validate researches. Due to diversed potential findings, the review utilized certain degree of limitations as criteria for selection. A multi-staging strategy is developed to identify significant and relevant review results.

Based from the analyses, literatures revealed that a good and comprehensive knowledge about HIV/AIDS transmission increases positive effects towards sexual behaviors and attitudes and is considered key step towards behavioral change. The findings assessed the degree of knowledge and disclosed that almost young adults (15-24 years old) had heard HIV/AIDS; however have misconceptions about the infection saying it is curable, doubtful on the effectiveness of educational programs, efficiency of the government to deliver services to the population concerned. Further, factors have been identified contributing in the low level of knowledge and awareness about the issue include perception of the risk factors associated with HIV, less access and safety concerns for not using condoms and contraception among others. Therefore, it is concluded that young people's knowledge must be assessed clearly to correct notion and myth about HIV/AIDS.
\end{abstract}

Keywords: HIV/AIDS, Knowledge, Awareness, Young Adult

\section{INTRODUCTION}

Despite the tremendous efforts of the World Health Organization (2001), health agencies worldwide and the mainstream media in their persistent objective of educating and warning the people of its detrimental effects of the infection, the cases continue to rise and remain to be unremitting.

Human Immunodeficiency Virus (HIV) has been tagged as a global problem and the deficiency of knowledge about its disease process, weak health care delivery system, risky sexual behavior, penury and economic fluctuation are the very reason why this has become a pandemic disease.

AIDS or widely known as Acquired Immune Deficiency Syndrome is caused by HIV that weakens the immune system, thus making the body vulnerable to opportunistic diseases like tuberculosis, pneumonia and other tumors that often lead to death. In the recent data from UNAIDS (2016) seventy-eight million people have become infected since the start of the epidemic in 1981.

The knowledge and prevention of HIV/AIDS have globally increased however, less than fifty percent of the young adults living in fifteen countries with the highest widespread presence cannot[JR1] correctly answer or even differentiate the basic queries regarding HIV, STD and its transmission.

Amidst evidence increased HIV-related knowledge among young adults, there are studies that revealed the resistance of openly discussing sexuality and sexrelated issues and health. Demographic health surveys reveal that highest rates of new HIV infection came from the tertiary school students.

There have been many researches explaining why there is an overwhelming increase of HIV infection among young adults and one of the notable reasons is that adolescents experience puberty at a very young age compared to the older generation.

There have been many studies about knowledge and risky sexual behaviors in Southeast Asia. However, few studies scientifically conducted to comprehensively asses the knowledge and behavior of young adults towards HIV/AIDS.

\section{Purpose and Statement of the Problem}

The aim of this analysis is to distinguish the degree of knowledge and awareness among young adults towards HIV/AIDS. The article would also [JR3] report the findings from a systematic review of studies identifying the misconceptions and myths about HIV/AIDS, thus endowing educational programs and execute appropriate intervention from the factual data gathered. 


\section{METHODOLOGY}

Due to the various potential findings, the review utilized a certain degree of limitation as criteria for selection. A multi-staging strategy was established to identify the relevant and significant review literatures. Key words such as HIV/AIDS, knowledge, awareness, young adults were utilized to retrieve significant literatures. EBSCO and Pro Quest databases were employed during [JR4] literature retrieval. The reviewer also limited the selection process by using considerable criteria.

Literatures [JR5] should be published from January 2016 to January 2018; respondents are young adults aging 15-24 years old (UNICEF, 2013) on the date of the research conduct; either male or female with or without educational background. The researcher could be a nurse, social worker, medical doctors or social scientist and conducted the study within the AsiaPacific countries. Literatures were excluded if it did not meet the inclusion criteria set by the reviewer.

\section{RESULTS AND DISCUSSION}

Based from the inclusion and exclusion criteria, relevant literatures were identified. There were 50, 294 literatures published from Pro Quest from 1948-2018 as per retrieval last June 24, 2018. From the total figure of publications, 11,603 of which were published from January 2015 to January 2018; 6,991 were considered peer reviewed scholarly journals. When the reviewer added keywords such as "young adults" and "Asia" the results were narrowed down to 891 .

The reviewer discovered that there were a lot of duplication in the studies and did not mention knowledge and awareness of HIV hence, only 260 literatures passed the criteria. These literatures were published in PLoS One (76); The Lancelet (39); AIDS and Behavior (34); BMC Public Health (31). Other literatures were also published under BMJ Open (13); BMC Health Services Research (10) and Journal of Economic Literature (10). However, there were only 25 researches wherein Asian countries served as research environment of the study. Themes common among the 25 researches are knowledge and awareness, myths and misconceptions of HIV/AIDS, management, educational program and government services

Based from the review, AIDS or widely known as Acquired Immune Deficiency Syndrome is caused by HIV that weakens the immune system, thus making the body vulnerable to opportunistic diseases like tuberculosis, pneumonia and other tumors that often lead to death. In the recent data from UNAIDS (2016) seventy-eight million people have become infected since the start of the epidemic in 1981.Human Immunodeficiency Virus (HIV) has been tagged as a global problem and the deficiency of knowledge about its disease process, weak health care delivery system, risky sexual behavior, penury and economic fluctuation are the very reason why this has become a pandemic disease

Comprehensive knowledge about HIV/AIDS transmission is very important in the prevention of its epidemic. Having good and comprehensive knowledge about its transmission increases positive effects towards sexual behaviors and attitudes and is considered to be a key step towards a person's behavioral change. The study is congruent with the finding of Tenkorang (2014) that comprehensive and good knowledge plays an important role as a requirement for warding off HIV/AIDS transmission and is a key factor for a young adult's behavioral change.

Comprehensive knowledge as defined by the United Nations Children's Emergency Fund (UNICEF, 2013) includes comprehending both condom utilization and restricting multiple sexual partners to one HIV seronegative partner were the best prevention strategies. Second is to increase awareness that a complete and healthy-looking person may have acquired the infection even if he does not look frail. Further, a decline of the most recurrent fallacy that HIV can be transmitted through mosquito or any other form vector bites and by eating with HIV infected person.

The Young Adults Fertility and Sexuality survey in 2013 assessed the degree of knowledge and awareness on certain issues like STI and HIV. The results of the survey yielded an almost universal awareness of HIV among young adolescents. Statistics in 2002 survey showed that $95.1 \%$ of the age bracket $15-24$ had heard of AIDS. On one hand, results also revealed three out of ten $(27.8 \%)$ young people who have misconceptions about the infection, and believe that the disease is curable. What is more alarming is that the figure significantly increased overtime.

The findings were further compounded by the fact that the results in 2016 survey, 73.4 percent of the young adults believed that they cannot contact the virus in the future. This apparent lack of progress in the accumulation of comprehensive knowledge about the infection raises doubts on the effectiveness of education programs, and the efficiency of the government's agenda to deliver these programs to the population concerned. 
Diverse cultural environments have revealed that comprehensive and adequate knowledge about HIV/AIDS often fall back behind the instantaneous shifts in sexual behaviors and activities. The factors that had been identified to contribute in the low level of comprehensive and unabridged HIV knowledge are the young adult's low perception of the risk factors associated with HIV, which leads to impediment sexual behavior. The unending and increasing inadequate education strategy as a scheme of the disease process and not keeping a positive health seeking behavior towards HIV/AIDS predisposed the young people to vulnerability.

The role of knowledge in addressing myth, and fallacies about HIV is fundamental in the cessation of misconceptions. The most common misunderstanding identified is that an HIV-infected person appears to be frail and ill and that the virus can be transmitted through a mosquito or any vector for that matter or by sharing food with an infected person. In sub-Saharan Africa, young adults between 15-24 years old harbor serious fallacies about HIV and how it was transmitted. In the study of Cohall et al.,(2001) he concluded that even though it is a common knowledge that mosquito doesn't transmit the virus from person to another, many people still believe that it's a good vector and vehicle for HIV transmission. Another misconception about HIV is that it can be transmitted whilst kissing an infected person. There were no evidences and researches that concluded saliva is one of the modes of transmitting the virus.

Despite facts that showed both adequate knowledge and attitudes towards risky sexual behavior are essential in combating HIV/AIDS epidemic, several studies around the globe also revealed gaps among young adult's perception about transmission, vulnerability or susceptibility and risky sexual behaviors towards HIV/AIDS infection (Majelantle et. al., 2014 ; Mkumbo, 2013). In their study, they concluded that misconceptions about the disease and its transmission continue to flourish among young adults. Supplying comprehensive information and continuous strategic HIV-knowledge relay is still pivotal in the fight against HIV/AIDS infection in order to completely remove the fallacies and misapprehensions about its transmission and to help these vulnerable young adults protect them from contracting it.

Comprehensive sex education programs by the government and private sectors in developed countries like the United States and developing countries like the Philippines showed that young adolescents who underwent sex education in schools, forums and conventions were significantly more likely to have greater knowledge and exhibit health promoting behaviors than those who did not receive the intervention. Young adults who underwent sex education programs have dramatically changed their sexual acts to delaying the onset of engaging sexual activities, limiting the frequency of intercourse, reducing the practice of several sexual partners, not practicing unprotected sex and increasing the use of contraceptives and condoms.

Other studies have backed previous literature regarding sex education that young adults with comprehensive and accurate knowledge about HIV prevention and its transmission are more likely to have a greater understanding about perilous sexual behaviors and feel they are more capable of reducing the risk of getting the infection. Although knowledge may not be enough in protecting these young adults, having comprehensive, accurate and complete information about HIV infection and its severity may affect young adult's self-efficacy thus leading to health promoting behaviors.

\section{CONCLUSION}

This systematic review is able to identify relative points to healthy sexual behavior through comprehensive knowledge and awareness towards HIV/AIDS. Comprehensive knowledge and awareness towards HIV/AIDS is essential to prevent acquiring the virus and from spreading it. While some studies have shown positive changes in sexual behavior associated with increased knowledge on HIV/AIDS, other studies have shown that people still practice unsafe sex despite having this knowledge.

The idea of increased knowledge resulting in safe sexual behavior aimed at reducing HIV infection and modifying or avoiding risky sexual behavior is at the core of most HIV/AIDS education campaigns. However, studies on the impact of knowledge on HIV on sexual behavior have been inconsistent, generating varied results. While some studies have shown positive changes in sexual behavior associated with increased knowledge on HIV/AIDS, other studies have shown that people still practice unsafe sex despite having this knowledge. Still other studies in diverse cultural settings yielded varied results.

Basic knowledge or awareness of HIV/AIDS does not seem to translate to modification of sexual behavior to favor safe sexual practices, as viewed by some researchers. Studies have shown a poor correlation between knowledge and sexual behavior, claiming that knowledge of HIV/AIDS seemed to be insufficient to deter people from practicing unsafe sex (Awoniyi, 2015). These studies have shown that high levels of 
awareness of HIV/AIDS exist among the youth population in their countries, yet their respondents acknowledged that they often had unsafe sex with highrisk partners.

The review recommends that the government should be stringent and firm in implementing the HIV program activities with regards to the prevention, and fight against stigma and discrimination. Furthermore, the review encourages HIV/AIDS organizations to actively use social media informatics to inventively reach out particular group of populations with targeted messages hence, delivering targeted and tailored educational methods to develop self-efficacy that is needed for long term behavioral changes.

\section{ACKNOWLEDGEMENT}

This work would not have been possible without the financial support of the Office of the Research and Development of Eastern Visayas State University of Ormoc City. I am especially indebted to Dr. Ma. Erenita V. Bahian, the Head of Research and Development of EVSU-OCC who have been supportive of my career goals and who worked actively to provide me with the protected academic time to pursue those goals.

I am grateful to all of those with whom I have had the pleasure to work during this and other related projects. Each of the members of my research group has provided me extensive personal and professional guidance and taught me a great deal about both scientific research and life in general. I would especially like to thank Professor Joel Ray Acob, the research coordinator of Visayas State University School of Nursing as my teacher and mentor, he has taught me more than I could ever give him credit for here. He has shown me, by his example, what a good nurse educator (and person) should be.

Nobody has been more important to me in the pursuit of this project than the members of my family. I would like to thank my parents, whose love and guidance are with me in whatever I pursue. They are the ultimate role models.

\section{REFERENCES}

Awoniyi, M. (2015). A Study of Nigerian Youths' Understanding, Perception Of HIV/AIDS Phenomenon and Sex Attitudes: The Link between Acceptance of Reality and Marketing Motives of Multinational Pharmceutical Companies.Journal of Service Science and Management, 8(4), pp 471-484 .

Cohall, A., Kassotis, J., Parks, R., Vaughan, R., Bannister, H. \& Northridge, M. (2001). Adolescents in the Age of AIDS: Myths, Misconceptions and Misunderstanding Regarding Sexually Transmitted Diseases. Journal of National Medical Association, 93(2), pp 64-69.

Majelantle, R., Keetile, M., Bainame, K., \& Nkawana, P. (2014). Knowledge, Opinions and Attitudes Towards HIV/AIDS Among Youth of Botswana. Journal of Global Economics, 2(1), Pages 7.

Mkumbo, K. (2013). Assessment of HIV/AIDS Knowledge, Attitudes and Behaviors among Students In Higher Education In Tanzania. Global Public Health, 8(10), pp 1168-1179.

Tenkorang, E.Y. (2014). Marriage, Widowhood, Divorce and HIV Risks among Women in SubSaharan Africa. International Health, 6 (1), pp $46-53$.

The Joint United Nations Programme on HIV/AIDS (UNAIDS).(2016).Fact Sheet November2016.Retrievedfrom:http://www.unaids.org/sites/default/fi les/media_asset/UNAIDS_FactSheet_en.pdf

UNICEF: United Nation Children's Fund. (2013). HIV and AIDS. Retrieved from: https://www.un.org/ youthenvoy/2013/09/unicef-the-united-nation-childrens-fund/

World Health Organization. (2001). Infections and infectious diseases A manual for nurses and midwives in the WHO European Region. Retrieved from: http://www.euro.who.int/_data/assets/pdf file/ 0013/102316/e79822.pdf 